\title{
NISTIR 5351
}

\section{Airborne Asbestos Method: Standard Test Method for Verified Analysis of Asbestos by Transmission Electron Microscopy - Version 2.0}

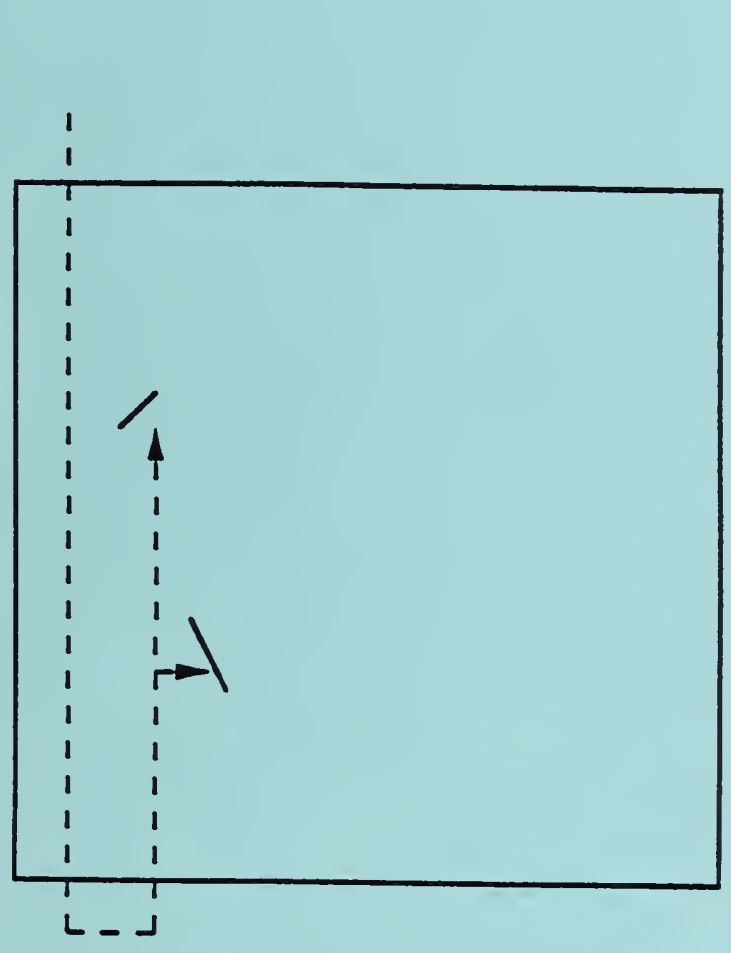

Shirley Turner

Eric B. Steel

U.S. DEPARTMENT OF COMMERCE

Technology Administration

National Institute of Standards and Technology

Nicroanalysis Research Group

Surface and Microanalysis Science Division Chemical Science \& Technology Laboratory Gaithersburg, MD 20899 



\title{
Airborne Asbestos Method: Standard Test Method for Verified Analysis of Asbestos by Transmission Electron Microscopy - Version 2.0
}

\author{
Shirley Turner \\ Eric B. Steel
}

U.S. DEPARTMENT OF COMMERCE

Technology Administration

National Institute of Standards

and Technology

Microanalysis Research Group

Surface and Microanalysis Science Division Chemical Science \& Technology Laboratory

Gaithersburg, MD 20899

March 1994

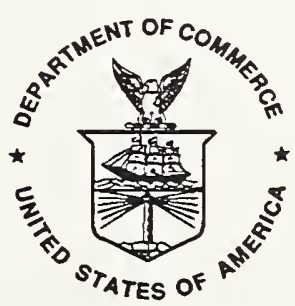

U.S. DEPARTMENT OF COMMERCE

Ronald H. Brown, Secretary

TECHNOLOGY ADMINISTRATION

Mary L. Good, Under Secretary for Technology

NATIONAL INSTITUTE OF STANDARDS

AND TECHNOLOGY

Arati Prabhakar, Director 



\section{Preface}

This Interagency Report (IR) is one of a series of IRs that will form the basis of a method for analysis of airborne asbestos by transmission electron microscopy. The form and style of the American Society for Testing and Materials (ASTM) was adopted as a standard format for this series of reports. 


\section{Scope}

1.1 This test method describes a procedure for verified analysis of asbestos by transmission electron microscopy.

1.2 The method is applicable only when sufficient information has been collected during the analyses of a grid square so that individual asbestos structures can be uniquely identified.

1.3 The method is written for the analysis of a grid square by two TEM operators but can be used for more than two operators with slight modifications. Due to the analysis of a grid square by more than one TEM operator, the test method can be applied only when contamination and beam damage of particles are minimized. The two TEM operators can use the same TEM for the analysis or the analyses can be done on different TEMs (in the same or in different laboratories).

1.4 The method can be used with any set of counting rules applied by all analysts. Though the method describes verification of asbestos particles, the method can also be used for verification of analyses of nonasbestos particles if all analysts use the same counting rules.

\section{Terminology}

2.1 Definitions:

2.1.1 TEM--transmission electron microscope.

2.1.2 grid square, grid opening--an area on a grid used for analysis of asbestos by transmission electron microscopy.

2.1.3 verified analysis--a procedure in which a grid opening is independently analyzed for asbestos by two or more TEM operators and in which a comparison and evaluation of the correctness of the analyses are made by a verifying analyst. Detailed information -- including absolute or relative location, a sketch, orientation, size (length, width), morphology, analytical information and identification -- is recorded for each observed structure.

2.1.3.1 Discussion--Verified analysis can be used to determine the accuracy of operators and to determine the nature of problems that the analyst may have in performing accurate analyses. Verified counts can be used to train new analysts and to monitor the consistency of analysts over time.

2.2 Description of Terms Specific to This Standard:

2.2.1 counting rules--rules used to determine the amount of asbestos present in an asbestos-containing sample. Counting rules are a part of most methods for analysis of asbestos by transmission electron microscopy including the AHERA method and the ISO method (see definitions below).

2.2.2 AHERA method ${ }^{n}$--procedure for analysis of asbestos by transmission electron microscopy developed by the Environmental Protection Agency with subsequent modifications by the National Institute of Standards and Technology.

2.2.3 ISO method ${ }^{2}$--procedure for analysis of asbestos by transmission electron microscopy developed by the International Standards Organization.

2.2.4 particle--an isolated collection of material deposited on a grid or filter.

2.2.5 structure--a particle or portion of a particle that contains asbestos and that is considered countable under the method used for asbestos analysis. A structure is a basic unit used in many methods of asbestos analysis to report the amount of asbestos present in a particle.

2.2.6 TEM operator, TEM analyst--person that analyzes a grid square by transmission electron microscopy to determine the presence of asbestos.

2.2.7 verifying analyst--person that compares the analyses of a grid square by two or more TEM operators. The reported asbestos is compared on a structure-by-structure basis by the verifying analyst. Structures that are not matched are relocated and reanalyzed by the verifying analyst. The verifying analyst is

\footnotetext{
${ }^{1}$ Code Fed. Reg. 1987, 52 (No. 210), 41826-41905.
}

${ }^{2}$ ISO 10312 1993, in press. 
preferably not one of the TEM operators. If this cannot be avoided, the job of verifying analyst should be rotated between the TEM operators.

2.2.8 TEM analysis form--form on which the analysis of a grid square is recorded. The information recorded for a verified analysis should include at least a sketch of the structure and information related to the absolute or relative location, size, identification and analytical data for the reported structures.

2.2.9 report form--form on which the evaluation of verified analyses is summarized. The form should be identical to or include all information given in Figure X1.1 of Appendix X1.

2.2.10 SR (structures reported)--the number of structures reported by a TEM analyst.

2.2.11 TP (true positive)--structure that is: 1) reported by both TEM operators or 2) reported by one operator and confirmed by the verifying analyst, or 3) reported by neither TEM operator but is found by the verifying analyst. The three types of true positives are discussed in the next three terms.

2.2.12 TPM (true positive-matched)--structure that is reported on the TEM analysis forms of both TEM operators.

2.2.12.1 Discussion--To qualify as a match, the structures should be comparable in the following characteristics: 1) absolute or relative location, 2) appearance in the sketch, 3) orientation, 4) size (length, width), 5) morphology (shape, hollow tube), 6) analytical information (chemistry and/or diffraction data), and 7) identification. In addition, the structures should be reported as countable by both analysts.

2.2.13 TPU (true postive-unmatched)--structure that is reported on the TEM analysis form of only one operator and that is confirmed as countable by the verifying analyst.

2.2.14 TPV (true positive found by verifying analyst)--structure not found by the two TEM operators but found by the verifying analyst.

2.2.15 TNS (total number of structures)--the number of structures determined to be in a grid opening by verified analysis of the grid opening. This value corresponds to the number of unique true positives found by the TEM operators and the verifying analyst.

2.2.15.1 Discussion--The value for the total number of structures is not necessarily the actual number on the grid square because both the TEM analysts and the verifying analyst may have missed one or more structures. The probability of a missed structure, however, decreases with an increased number of analysts.

2.2.16 FN (false negative)--structure that has not been reported as countable by one of the TEM analysts. False negatives can be divided into two categories-type $\mathrm{A}$ and type $\mathrm{B}$ as discussed in the next two terms.

2.2.17 FNA (false negative-type A)--false negative that was recorded on a TEM analyst's TEM analysis form but not reported as a structure. Some reasons for this type of false negative include: 1) structure misidentified as nonasbestos, 2) confusion with the counting rules, 3) incorrect length determination.

2.2.18 FNB (false negative-type B)--false negative that was not recorded on a TEM analyst's TEM analysis form. A reason for this type of false negative is that a structure was missed by an analyst.

2.2.19 FP (false positive)--reported particle that is incorrectly identified as a structure. Some reasons for false positives include: 1) structures counted more than one time, 2) materials misidentified as asbestos, 3) confusion with the counting rules, 4) incorrect length determination.

2.2.20 TN (true negative)--reported particle that is correctly characterized as zero structures.

2.2.21 NL (not located structure)--structure reported on one TEM analyst's TEM analysis form that cannot be located by the verifying analyst.

2.2.21.1 Discussion--The value for NL should be zero for most verified analyses, especially if the grid has not been removed from the TEM between the two analysts' counts. If, however, a grid has been removed from an instrument, there is a small possibility of fiber loss.

2.2.22 $A M B$ (ambiguous structure)--a structure that 1 ) is identified as a structure by only one TEM operator and 2) is found by the verifying analyst but cannot be unambiguously identified as a structure due to beam damage, contamination, or other factors. 


\section{Significance and Use}

3.1 The analysis of asbestos by transmission electron microscopy is important for the determination of the cleanliness of air or water and for research purposes. Verified analyses provide more accurate values for the concentration of asbestos on a grid opening than obtained by other methods. The accuracy should increase with an increased number of analysts participating in the verified count.

3.2 The test method can be used as part of a quality assurance program for asbestos analyses and as a training procedure for new analysts. The values for TP/TNS and FP/TNS can be plotted $v s$ time on control charts to show improvements or degradations in the quality of the analyses. Experienced analysts should attain TP/TNS values $\geq 0.85$ and FP/TNS values $\leq 0.05$. The test method can be used to characterize the types and, in many cases, the causes of problems experienced by TEM analysts.

3.3 The average of values obtained for TP/TNS and FP/TNS can be used to determine the analytical uncertainty for routine asbestos analyses.

\section{Procedure}

NOTE 1-- This test method involves two TEM operators and a verifying analyst. The steps discussed in items 4.1 and 4.2 are to be followed by the person coordinating the analyses by the TEM operators. This person can be one of the TEM operators, the verifying analyst or an independent person (e.g., a quality assurance officer). The steps discussed starting with item 4.3 are to be followed by the verifying analyst.

4.1 Obtain analyses of a grid square for asbestos by two TEM operators. Conduct the analyses independently so that the second operator has no knowledge of the results obtained by the first operator.

4.1.1 Require that the TEM operators record on the TEM analysis form information related to the absolute location of the structures or conduct analyses so that the relative location of the structures can be compared.

NOTE 2-- The absolute location of the structures can be recorded by various means including use of a digital voltmeter or computer readable stepping motors to record the position of a structure. To preserve information about the relative location of the reported structures, the analyses must be conducted so that both analysts: 1) orient the grid in the TEM in the same fashion, 2) start the analysis from the same corner of the grid square, 3) initially scan in the same direction, and 4) scan the grid square in parallel traverses.

4.1.2 Require that the TEM operators record on the TEM analysis form a sketch of the structure, the dimensions of the structure, analytical data and whether the structure is countable. The sketch of the structure should include any nearby features that could aid in subsequent identification - for instance, nearby particles, sample preparation features or grid bars.

4.2 Submit the analyses of the two TEM operators to the verifying analyst.

NOTE 3-- The remainder of this section describes procedures to be followed by the verifying analyst. The procedure for comparison of the TEM analysis forms is given in items 4.3-4.6 and examples of comparisons of count sheets are given in Figs. X2.1-X2.9 of Appendix 2. Appendix 3 contains a summary of the comparison process (Fig. X3.1) and a flow chart for comparison of structures in the TEM (Fig. X3.2). The procedure for completion of the report form is given in item 4.7 .

4.3 Compare the two TEM analysis forms on a structure-by-structure basis. If a match of asbestos structures is observed, label both sketches with a TPM(number) either in the sketch box or in a column specifically designated for verified counts. An example is given in Fig. X2.1 of Appendix X2.

NOTE 4-- The next step in the procedure (item 4.4) is optional. The most prudent approach is to examine unmatched structures in the TEM (item 4.5). 
4.4 Determine if the status of any of the unmatched structures can be unambiguously decided by examining the TEM analysis forms. If there is ambiguity in determining the status of a structure, the verifying analyst must examine the structure in the TEM as described in items 4.5-4.6. The comparison of TEM analysis forms and labelling of unmatched structures can be relatively straight foward as shown in Fig. $\mathrm{X} 2.2$ - X2.4 of Appendix X2 or more complex as described in the next item.

4.4.1 For most cases, the identification of true positives, false positives and false negatives can be done on a structure-by-structure basis. This cannot be done, however, in cases where analysts determine different numbers of countable structures in an asbestos-containing particle. In such cases, both analysts should be assigned one TPM(number) for identifying the particle as containing countable asbestos. The remaining structures are assigned TPU, FP or FN depending on the particular situation. Examples of such cases are given in Fig. X2.5 and Fig. X2.6 of Appendix X2.

4.5 Determine the status of any remaining unlabelled structures by examining the grid square in the TEM. Examples of TEM analysis forms containing structures that must be examined by transmission electron microscopy are given in Figs. X2.7 - X2.9 of Appendix 2. For each unlabelled structure requiring examination by transmission electron microscopy, follow items 4.5.1-4.5.7 and 4.6 until the structure is labelled. If there is another unlabelled structure, go back to item 4.5.1 and repeat the procedure. Continue until all structures are labelled. A summary flow chart for examination by TEM is given in Fig. X3.2. The procedure and flowchart do not cover the counting discrepancy discussed in item 4.4.1. If such a situation is recognized, the verifying analyst should follow the procedure given in item 4.4.1 and in the examples in Figs. $\mathrm{X} 2.5$ and X2.6.

NOTE 5-- The procedure in items 4.5.1-4.5.7 should cover the great majority of cases encountered when attempting to determine the status of the structures. There may, however, be more complex situations not covered in the procedure. If so, the verifying analyst should apply the basic principles outlined in items 4.5.14.5.7 and 4.4.1.

4.5.1 Determine if the reported structure can be located. If the structure cannnot be found, label the reported structure NL (place the label next to the sketch or in a column specifically designated for verified analyses).

4.5.2 If the reported structure is found, determine if a judgement can be made as to its countability. If the structure cannot be judged as to its countability due to beam damage, contamination or other factors, label the reported structure AMB.

4.5.3 If a judgement can be made as to the countability of the reported structure, determine if the structure is countable. If the reported structure is not countable, label it FP(number). A unique number is given to the FP label so that it can be specifically referred to in the report form. Optional: Check the other analyst's TEM analysis form. If the other analyst sketched the particle and correctly reported it as noncountable, label the particle TN(number). Note: The values for TN are not recorded on the report form.

4.5.4 If the reported structure is correctly identified as a structure, determine if it was reported as countable elsewhere on the same analyst's TEM analysis form (i.e., the analyst counted the structure twice). If it is a duplicate, label the reported structure FP(number).

4.5.5 If the reported structure is not a duplicate, label the structure TPU(number).

4.5.6 Determine if the other TEM operator recorded a sketch of the structure. If the other TEM operator did not report the structure on his/her TEM analysis form, place an FNB(number) on their TEM analysis form in the approximate location where the structure should have been found. The number should correspond to that given to the TPU on the first analyst's TEM analysis form.

4.5.7 If the other TEM operator recorded a sketch of the structure, label the sketch with an FNA(number). The number should correspond to that given to the TPU on the first analyst's TEM analysis form.

4.6 Countable asbestos structures reported by neither TEM operator but found by the verifying analyst in the course of examining a grid square should be recorded on a separate TEM analysis form and labelled 
TPV(number). The TEM operators should be assigned an FNA(number) or FNB(number) as described in items 4.5.6-4.5.7.

4.7 Complete the report form as described in items 4.7.1-4.7.10.

4.7.1 Complete the heading of the report form and fill in the initials or names of the two TEM operators on the first line of the report form table.

4.7.2 Count the number of asbestos structures obtained by each analyst and enter the value as SR (structures reported) on the report form.

4.7.3 Determine the number of true positives that are matched (TPM), the number of true positives that are unmatched (TPU) and the total number of true positives (TP) obtained for each TEM operator on the grid square and enter the values on the report form.

4.7.4 Determine and record on the report form the number of true positives found by the verifying analyst (TPV).

4.7.5 Determine and record on the report form the total number of structures (TNS) on the grid square.

4.7.6 Determine and record on the report form for each operator the following: 1) the number of false positives (FP), 2) the number of false negatives (FN), 3) the number of false negatives of type A and type $B$ (FNA, FNB), 4) the number of structures that were not located (NL) and 5) the number of ambiguous structures (AMB).

4.7.7 Determine and record the values for TP/TNS, FP/TNS to two decimal places.

4.7.8 List on the report form the suspected reasons for the false positives obtained by each analyst. Some examples would be as follows: incorrect length measurement, structures counted twice, problem with interpretation of the counting rules, misidentification of a structure.

4.7.9 List on the report form the suspected reasons for false negatives (FNA and FNB). Some examples would be: incorrect length measurement, problem with interpretation of the counting rules, misidentification of material as asbestos, possible loss of sense of direction, and insufficient overlap of traverses.

4.7.10 Append any other relevant comments to the report form (quality of the preparation, etc.).

4.8 Check the numbers on the report form using the equations given in the calculation section.

\section{Calculation}

5.1 The values on the report form should be consistent with the following equations:

For both analyses:

$\mathrm{TNS}=\mathrm{TPM}+\mathrm{TPU}($ Operator 1$)+\mathrm{TPU}($ Operator 2$)+\mathrm{TPV}$

For a given analysis:

$$
\begin{aligned}
& \mathrm{SR}=\mathrm{TP}+\mathrm{FP}+\mathrm{NL}+\mathrm{AMB} \\
& \mathrm{TP}=\mathrm{TPM}+\mathrm{TPU} \\
& \mathrm{FN}=\mathrm{FNA}+\mathrm{FNB} \\
& \mathrm{TNS}=\mathrm{TP}+\mathrm{FN} \\
& 1=\mathrm{TP} / \mathrm{TNS}+\mathrm{FN} / \mathrm{TNS}
\end{aligned}
$$




\section{Precision and Bias}

6.1 To determine the precision of the method, independent verified analyses were conducted by operators in two laboratories on a set of 21 grid squares. The mean value for TNS for the data set was 16.2 structures/grid square and the pooled standard deviation of the pairs of verified count determinations was 1.12 structures/grid square. The confidence at approximately the $95 \%$ level ( 2 standard deviations) of a reported verified count value in this data set is 2.24 structures/grid square or $13.9 \%$ of the mean value for TNS. We use $13.9 \%$ as an estimate of the imprecision of the method.

NOTE 6-- The differences in the values obtained for the independent verified analyses described in item 6.1 are, for the most part, due to differences in interpretation of the counting rules. The structures analyzed in the study were complex and therefore the imprecision estimate discussed above likely represents an upper bound to the imprecision for the method.

6.2 The bias in the method will vary depending upon interpretation of the counting rules used in the analysis by the TEM operators and verifying analyst.

\section{Keywords}

7.1 asbestos; quality assurance; transmission electron microscopy; verified analysis 


\section{APPENDIXES}

\section{(Nonmandatory Information)}

\section{X1. TEST REPORT FORM}

Fig. X1.1 The following format is suggested for use by the verifying analyst to report the comparison of the TEM operators' TEM analysis forms.

Grid box:

Date:

Grid slot:

Verifying Analyst:

Grid square:

\begin{tabular}{||l|l|l||}
\hline & Analysis 1 & Analysis 2 \\
\hline \hline TEM Operator & & \\
\hline Structures Reported (SR) & & \\
\hline True Positives (TP) & & \\
\hline${ }^{* T P M}$ & & \\
\hline TPU & & \\
\hline${ }^{* T P V}$ & & \\
\hline${ }^{*}$ Total \# Structures (TNS) & & \\
\hline False Positives (FP) & & \\
\hline False Negatives (FN) & & \\
\hline FNB & & \\
\hline FNB & & \\
\hline Not Located (NL) & & \\
\hline Ambiguous (AMB) & & \\
\hline TP/TNS & & \\
\hline FP/TNS & & \\
\hline \hline
\end{tabular}

*The values for these items will be the same for both analyses. 
Test Report Form (continued)

1) List details of suspected reasons for false positives. For each analyst describe reasons for FP1, FP2, FP3, etc. Note - it may not be possible to determine the reason for false positives for some structures.

2) List details of suspected reasons for false negatives (type $A$ and type $B$ ). For each analyst describe reasons for FNA1, FNA2, etc.; FNB1, FNB2, etc. Note - it may not be possible to determine the reasons for false negatives for some structures. 


\section{X2. EXAMPLES OF COMPARISONS OF TEM ANALYSIS FORMS}

[Note: The TEM analysis forms shown in the examples are abbreviated and do not contain analysis information. The AHERA counting rules (1987) were used for all analyses.]

Analyst 1

\begin{tabular}{|c|c|c|c|c|c|}
\hline 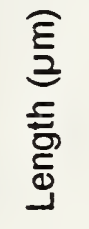 & 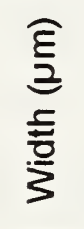 & Sketch & 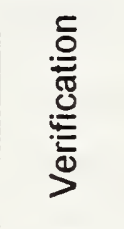 & 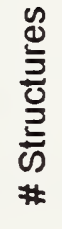 & $\underline{\underline{ }}$ \\
\hline 1.3 & 0.1 & & TPM1 & 1 & $\mathrm{Chr}$ \\
\hline 0.7 & 0.1 & & TPM2 & 1 & Chr \\
\hline 1.0 & 0.1 & & TPM3 & 1 & $\mathrm{Chr}$ \\
\hline
\end{tabular}

Analyst 2

\begin{tabular}{|c|c|c|c|c|c|}
\hline 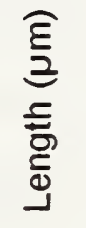 & 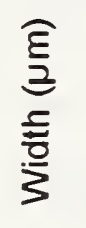 & Sketch & 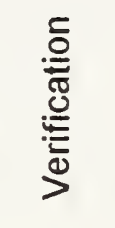 & 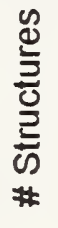 & $\underline{\underline{ }}$ \\
\hline 1.3 & 0.1 & & TPM1 & 1 & Chr \\
\hline 1.0 & 0.1 & & TPM3 & 1 & Chr \\
\hline 0.7 & 0.1 & & TPM2 & 1 & Chr \\
\hline
\end{tabular}

Fig. X2.1 Example of matching structures on two TEM analysis forms (refer to item 4.3 of the procedure). Three structures on a grid square were found by both analysts. The relative order of the last two structures is different on the two TEM analysis forms; this may be due to the nature of the traverses by the analysts.

Matching structures are indicated by TPM(number). 
Analyst 1

\begin{tabular}{|c|c|c|c|c|c|}
\hline 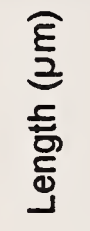 & $\begin{array}{l}\widehat{\frac{\hat{E}}{2}} \\
\text { 产 } \\
\frac{\bar{t}}{3}\end{array}$ & Sketch & 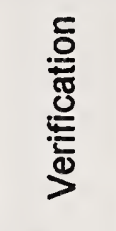 & 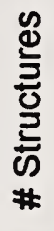 & 으 \\
\hline 1.3 & 0.1 & & TPM1 & 1 & $\mathrm{Chr}$ \\
\hline 0.7 & 0.1 & & TPM2 & 1 & $\mathrm{Chr}$ \\
\hline 1.0 & 0.1 & & TPM3 & 1 & $\mathrm{Chr}$ \\
\hline 0.7 & 0.1 & & FP1 & 1 & $\mathrm{Chr}$ \\
\hline
\end{tabular}

Analyst 2

\begin{tabular}{|c|c|c|c|c|c|}
\hline 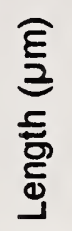 & 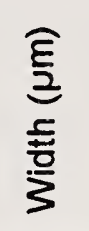 & Sketch & 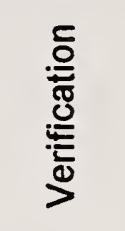 & 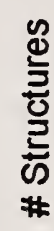 & 으 \\
\hline 1.3 & 0.1 & & TPM1 & 1 & $\mathrm{Chr}$ \\
\hline 1.0 & 0.1 & & TPM3 & 1 & $\mathrm{Chr}$ \\
\hline 0.7 & 0.1 & & TPM2 & 1 & $\mathrm{Chr}$ \\
\hline & & & & & \\
\hline & & & & & \\
\hline
\end{tabular}

Fig. X2.2 Example of determining the status of an unmatched structure from TEM analysis forms (refer to item 4.4 of the procedure). Three of the structures match in the two analyses. The last structure of analyst 1 is unmatched but can be seen from the TEM analysis form to be a duplicate of the second structure obtained by the same analyst (the two structures have the same identification, dimensions, orientation and a similar nearby particle). The duplicate structure is therefore assigned an FP1. 
Analyst 1

\begin{tabular}{|c|c|c|c|c|c|}
\hline $\begin{array}{l}\widehat{\underline{\xi}} \\
\text { 言 } \\
\text { 駦 }\end{array}$ & $\begin{array}{l}\widehat{\frac{\xi}{3}} \\
\frac{5}{5} \\
\text { 咅 }\end{array}$ & Sketch & 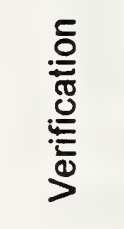 & 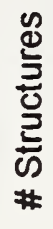 & 으 \\
\hline 0.6 & 0.1 & & TPU1 & 1 & $\mathrm{Chr}$ \\
\hline
\end{tabular}

Analyst 2

\begin{tabular}{|c|c|c|c|c|c|}
\hline 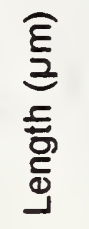 & 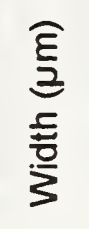 & Sketch & 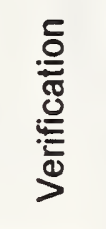 & 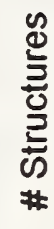 & 으 \\
\hline 0.6 & 0.1 & & FNA1 & 0 & $\mathrm{Chr}$ \\
\hline
\end{tabular}

Fig. X2.3 Example of determining the status of unmatched structures from TEM analysis forms (refer to item 4.4 of the procedure). Both analysts have found the same particle as indicated by the dimensions, identification and orientation of the structure. However, analyst 2 has reported that the particle is not a structure (the cause of this oversight is not known). Analyst 1 is assigned a TPU1 and analyst 2 an FNA1. 


\section{Analyst 1}

\begin{tabular}{|c|c|c|c|c|c|}
\hline 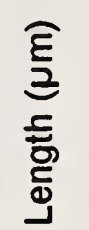 & 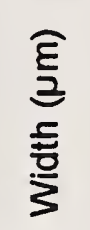 & Sketch & 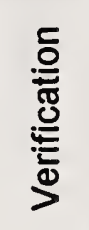 & 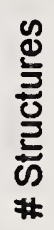 & 으 \\
\hline 0.4 & 0.1 & & FP1 & 1 & $\mathrm{Chr}$ \\
\hline
\end{tabular}

Analyst 2

\begin{tabular}{|c|c|c|c|c|c|}
\hline $\begin{array}{l}\widehat{\bar{\xi}} \\
\text { 咅 } \\
\text { 产 }\end{array}$ & $\begin{array}{l}\hat{\frac{\xi}{2}} \\
\frac{5}{\frac{5}{3}} \\
\frac{7}{3}\end{array}$ & Sketch & 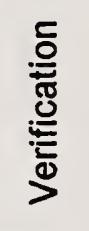 & 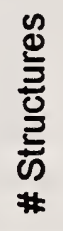 & 은 \\
\hline 0.4 & 0.1 & & TN1 & 0 & $\mathrm{Chr}$ \\
\hline
\end{tabular}

Fig. X2.4 Example of determining the status of unmatched structures from TEM analysis forms (refer to item 4.4 of the procedure). Both analysts have found the same particle as indicated by the dimensions, identification and orientation of the particle on both TEM analysis forms. However, analyst 1 has reported that the particle is a structure (the cause of this oversight is not known). Analyst 1 is assigned an FP1 and analyst 2 a TN1. 


\section{Analyst 1}

\begin{tabular}{|c|c|c|c|c|c|}
\hline 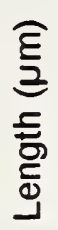 & 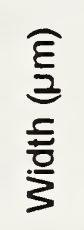 & Sketch & 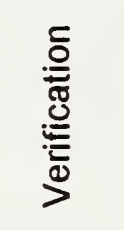 & 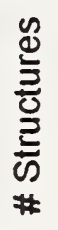 & 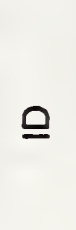 \\
\hline 1 & 0.6 & & & 1 & Chr \\
\hline & & & & & \\
\hline & & & & & \\
\hline & & & & & \\
\hline & & & & & \\
\hline
\end{tabular}

Analyst 2

\begin{tabular}{|c|c|c|c|c|c|}
\hline 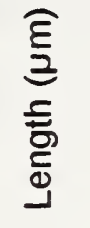 & 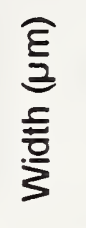 & Sketch & 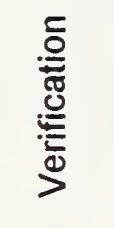 & 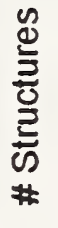 & 으 \\
\hline & & & & & \\
\hline 1 & 0.1 & $\mathrm{~F} 1$ & TPM1 & 1 & Chr \\
\hline 0.6 & 0.1 & F2 & TPU1 & 1 & Chr \\
\hline
\end{tabular}

Fig. X2.5 Example of determining the status of unmatched structures from TEM analysis forms (refer to item 4.4.1 of the procedure). Both analysts have found the same asbestos-containing particle as indicated by the dimensions, identification, and orientation of the particle. However, analyst 1 has reported one countable structure and analyst 2 has reported two countable structures. Under the AHERA counting rules, analyst 2 is correct. The structure reported by analyst 1 is assigned both a TPM1 and an FNA1. The two structures reported by analyst 2 are assigned a TPM1 and a TPU1, respectively. 
Analyst 1

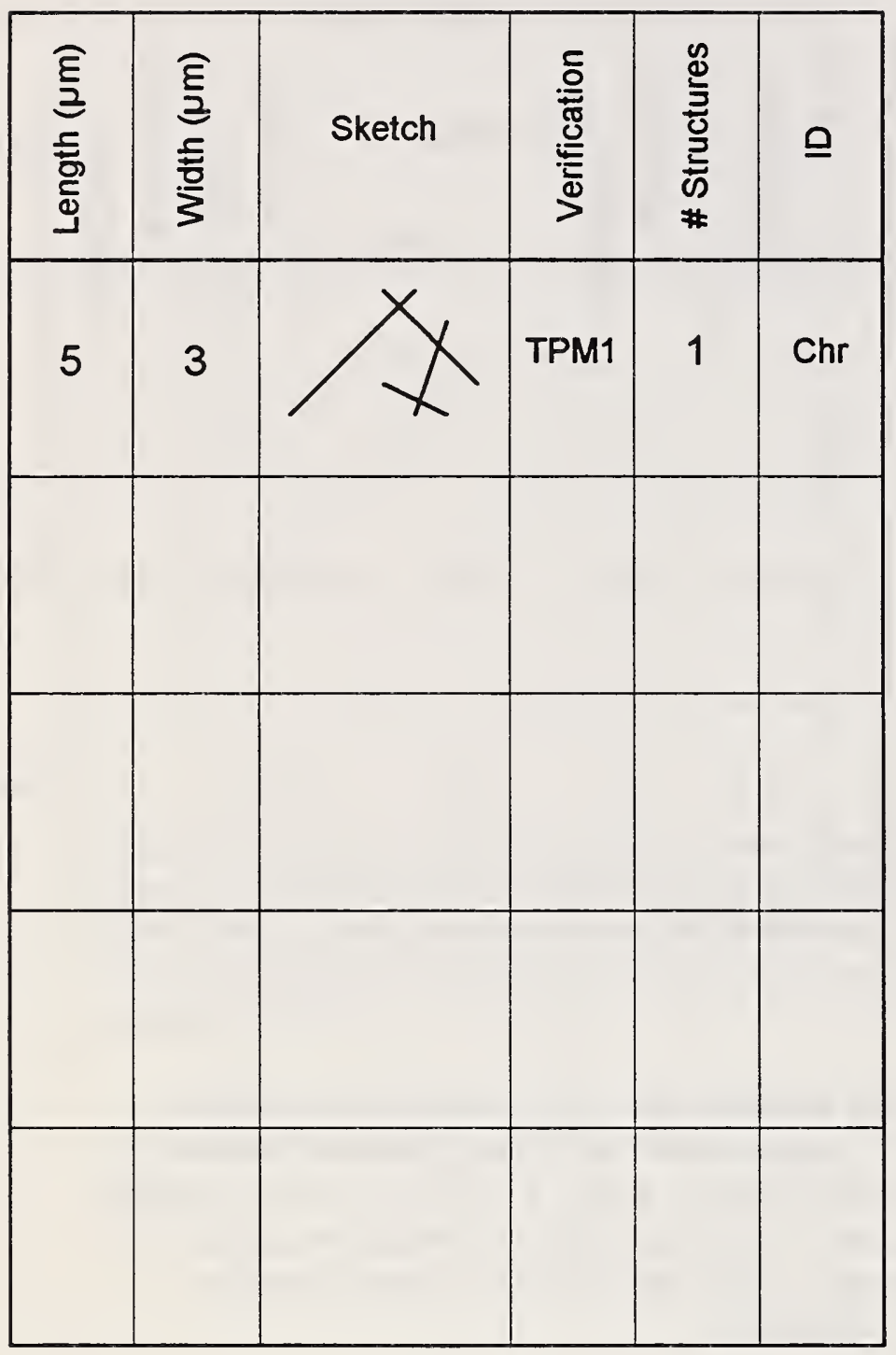

Analyst 2

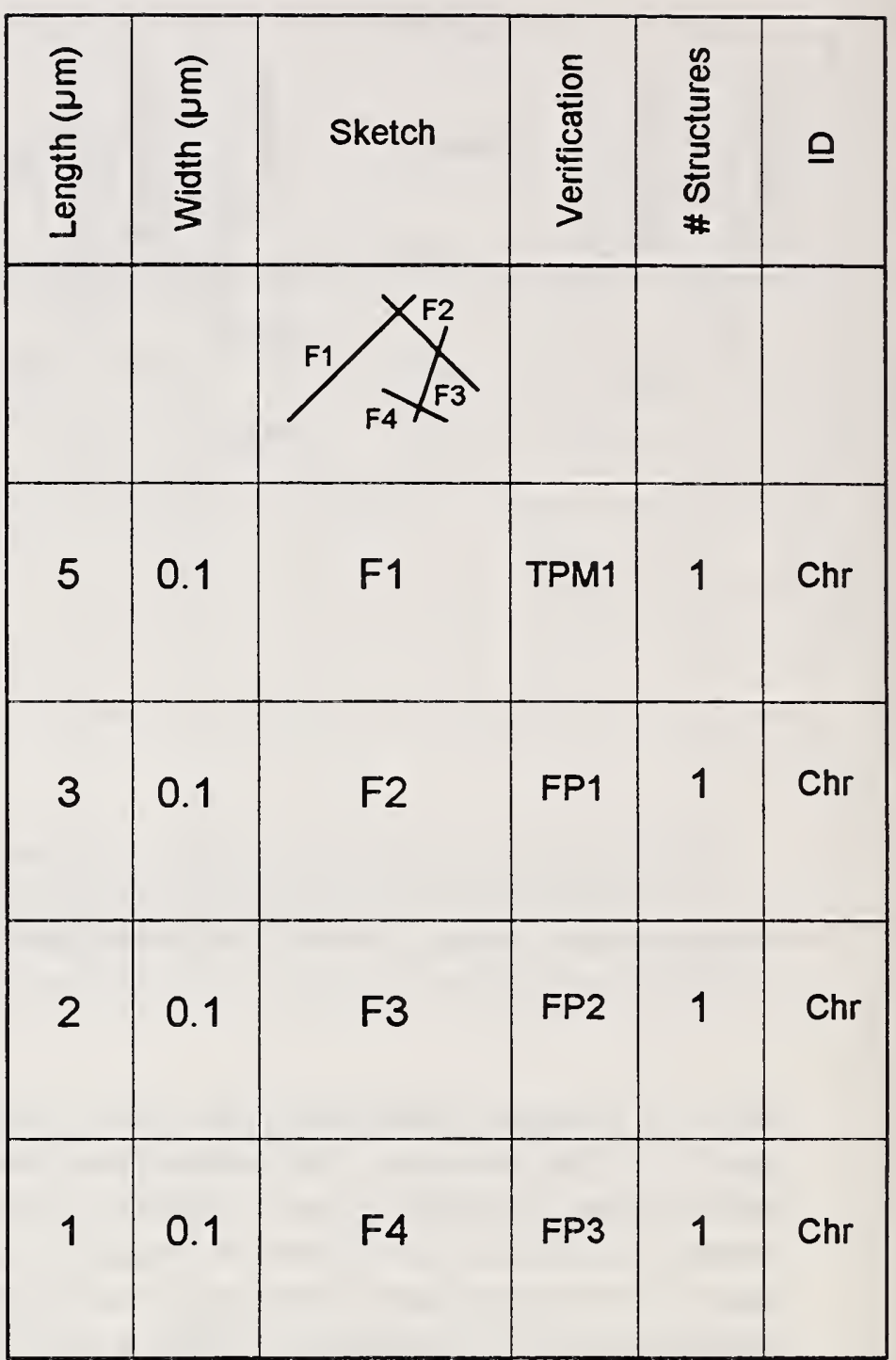

Fig. X2.6 Example of determining the status of unmatched structures from TEM analysis forms (refer to item 4.4.1 of the procedure). Both analysts have found the same asbestos-containing particle as indicated by the dimensions, identification, and orientation of the particle. However, analyst 1 has reported one structure and analyst 2 has reported four structures. Under the AHERA counting rules, analyst 1 is correct. The structure reported by analyst 1 is assigned a TPM1. The first structure reported by analyst 2 is labelled TPM1 and the remaining three reported structures are labelled FP1-FP3. 


\begin{tabular}{|c|c|c|c|c|c|}
\hline 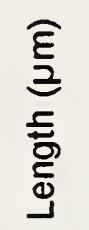 & 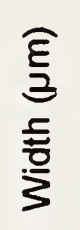 & Sketch & 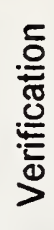 & 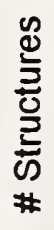 & 으 \\
\hline 0.4 & 0.1 & & & 0 & Chr \\
\hline
\end{tabular}

\begin{tabular}{|c|c|c|c|c|c|}
\hline 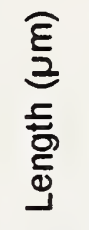 & 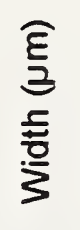 & Sketch & 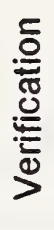 & 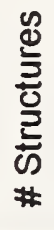 & 으 \\
\hline 0.6 & 0.1 & & & 1 & Chr \\
\hline
\end{tabular}

a

\begin{tabular}{|c|c|c|c|c|c|}
\hline 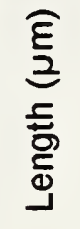 & 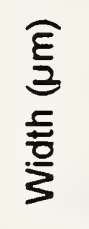 & Sketch & 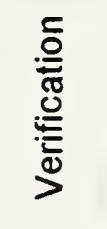 & 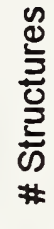 & 으 \\
\hline 0.4 & 0.1 & & FNA1 & 0 & $\mathrm{Chr}$ \\
\hline
\end{tabular}

\begin{tabular}{|c|c|c|c|c|c|}
\hline 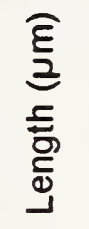 & 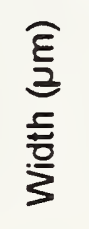 & Sketch & 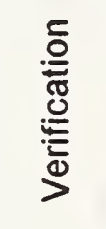 & 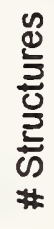 & 으 \\
\hline 0.6 & 0.1 & & TPU1 & 1 & $\mathrm{Chr}$ \\
\hline
\end{tabular}

b

\begin{tabular}{|c|c|c|c|c|c|}
\hline 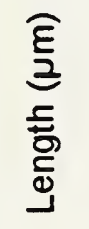 & 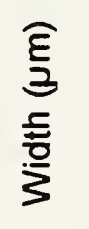 & Sketch & 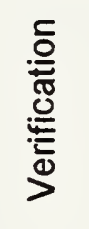 & 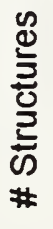 & 으 \\
\hline 0.4 & 0.1 & & TN1 & 0 & $\mathrm{Chr}$ \\
\hline
\end{tabular}

\begin{tabular}{|c|c|c|c|c|c|}
\hline 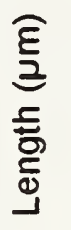 & $\begin{array}{l}\widehat{\hat{\varepsilon}} \\
\frac{1}{2} \\
\frac{\bar{z}}{3}\end{array}$ & Sketch & 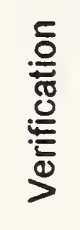 & 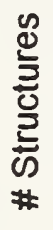 & 으 \\
\hline 0.6 & 0.1 & & FP1 & 1 & $\mathrm{Chr}$ \\
\hline
\end{tabular}

Fig. X2.7 Example of unmatched structures that must be examined by TEM (refer to item 4.5 of the procedure). a) Both analysts have likely found the same asbestos-containing particle as indicated by the identification and orientation of the fiber and by the presence of a similar particle nearby. However, the dimensions reported by the analysts differ and analyst 1 has reported zero structures and analyst 2 has reported one structure. The verifying analyst should determine the correct length of the fiber and determine if it qualifies as a structure. b) One possible outcome is that the verifying analyst finds that analyst 2 is correct. Analyst 2 is assigned a TPU1 and analyst 1 an FNAl. c) A second possible outcome is that the verifying analyst finds that analyst 2 is correct. Analyst 1 is assigned a TN1 and analyst 2 an FP1. 


\section{Analyst 1}

\begin{tabular}{|c|c|c|c|c|c|}
\hline 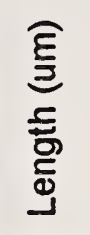 & 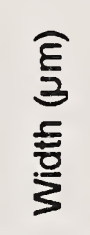 & Sketch & 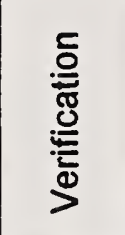 & 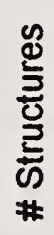 & 으 \\
\hline 1.3 & 0.1 & & TPM1 & 1 & $\mathrm{Chr}$ \\
\hline 0.6 & 0.1 & & & 1 & $\mathrm{Chr}$ \\
\hline 1.0 & 0.1 & & TPM2 & 1 & $\mathrm{Chr}$ \\
\hline
\end{tabular}

Analyst 2

\begin{tabular}{|c|c|c|c|c|c|}
\hline 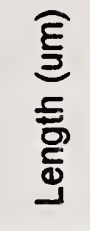 & $\begin{array}{l}\widehat{\frac{E}{2}} \\
\frac{5}{\bar{t}} \\
\frac{\bar{y}}{3}\end{array}$ & Sketch & 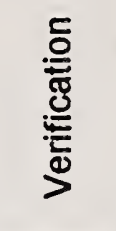 & 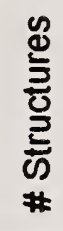 & 은 \\
\hline 1.3 & 0.1 & & TPM1 & 1 & $\mathrm{Chr}$ \\
\hline 1.0 & 0.1 & & TPM2 & 1 & $\mathrm{Chr}$ \\
\hline & & & & & \\
\hline & & & & & \\
\hline
\end{tabular}

Fig. X2.8 Example of unmatched structures that must be examined by TEM (refer to item 4.5 of the procedure). a) Analyst 1 has reported one structure that analyst 2 has not reported. The verifying analyst should attempt to find the particle and determine if it qualifies as a structure. b) One possible outcome is that the verifying analyst finds that analyst 1 is correct. Analyst 1 is assigned a TPUl and analyst 2 is assigned an FNB1. c) Another possible outcome is that the reported structure is not located. Analyst 1 is assigned an NL. Other possibilities (not illustrated) are that analyst 1 is incorrect (the particle is then labelled FP) or that the structure is too contaminated for characterization (the particle is then labelled AMB). 
Analyst 1

\begin{tabular}{|c|c|c|c|c|c|}
\hline $\begin{array}{l}\widehat{\hat{g}} \\
\text { 禀 } \\
\text { 离 }\end{array}$ & $\begin{array}{l}\widehat{\hat{\varepsilon}} \\
\frac{2}{2} \\
\frac{\hat{y}}{3}\end{array}$ & Sketch & 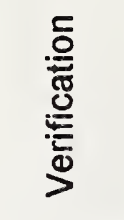 & 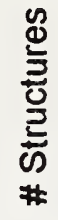 & 으 \\
\hline 1.3 & 0.1 & & TPM1 & 1 & $\mathrm{Chr}$ \\
\hline 0.6 & 0.1 & & TPU1 & 1 & $\mathrm{Chr}$ \\
\hline 1.0 & 0.1 & & TPM2 & 1 & Chr \\
\hline
\end{tabular}

Analyst 2

\begin{tabular}{|c|c|c|c|c|c|}
\hline 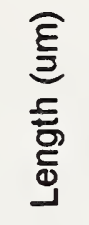 & 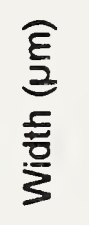 & Sketch & 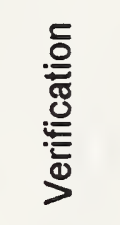 & 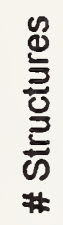 & $\underline{\underline{ }}$ \\
\hline 1.3 & 0.1 & & TPM1 & 1 & $\mathrm{Chr}$ \\
\hline 1.0 & 0.1 & & TPM2 & 1 & $\mathrm{Chr}$ \\
\hline & & & & & \\
\hline & & & & & \\
\hline
\end{tabular}

b

\begin{tabular}{|c|c|c|c|c|c|}
\hline $\begin{array}{l}\widehat{\hat{\xi}} \\
\text { 言 } \\
\text { 离 }\end{array}$ & $\begin{array}{l}\hat{\frac{\xi}{2}} \\
\text { 产 } \\
\text { 产 }\end{array}$ & Sketch & 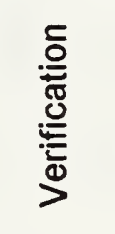 & 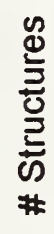 & ㅇ \\
\hline 1.3 & 0.1 & & TPM1 & 1 & $\mathrm{Chr}$ \\
\hline 0.6 & 0.1 & & NL1 & 1 & $\mathrm{Chr}$ \\
\hline 1.0 & 0.1 & & TPM2 & 1 & $\mathrm{Chr}$ \\
\hline
\end{tabular}

\begin{tabular}{|c|c|c|c|c|c|}
\hline $\begin{array}{l}\widehat{\hat{E}} \\
\text { 言 } \\
\text { 产 }\end{array}$ & 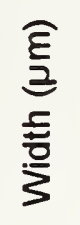 & Sketch & 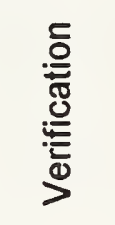 & 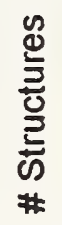 & $\underline{\underline{ }}$ \\
\hline 1.3 & 0.1 & & TPM1 & 1 & Chr \\
\hline 1.0 & 0.1 & & TPM2 & 1 & Chr \\
\hline & & & & & \\
\hline & & & & & \\
\hline
\end{tabular}

Fig. X2.8 (caption on previous page). 
Analyst 1

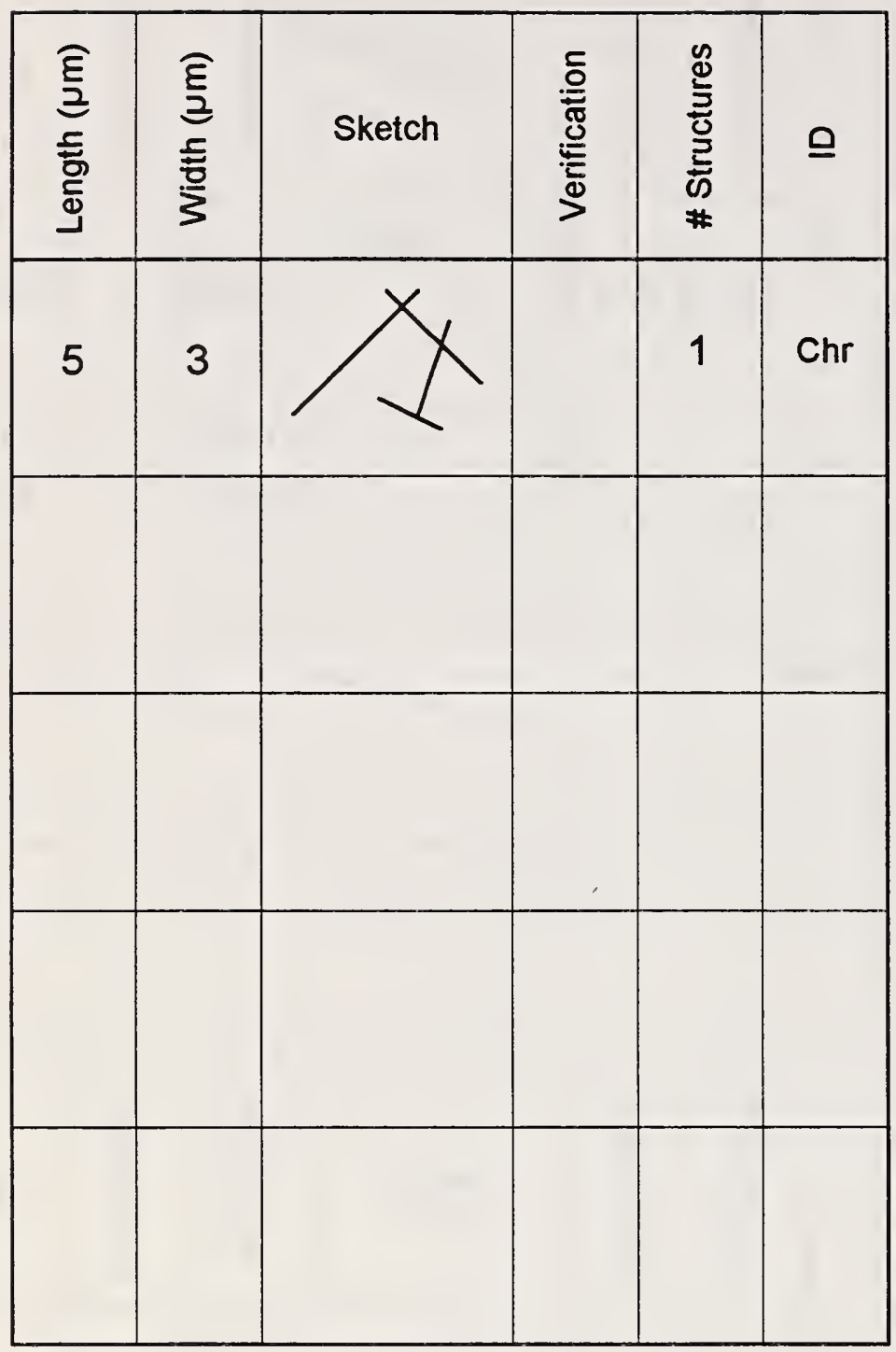

Analyst 2

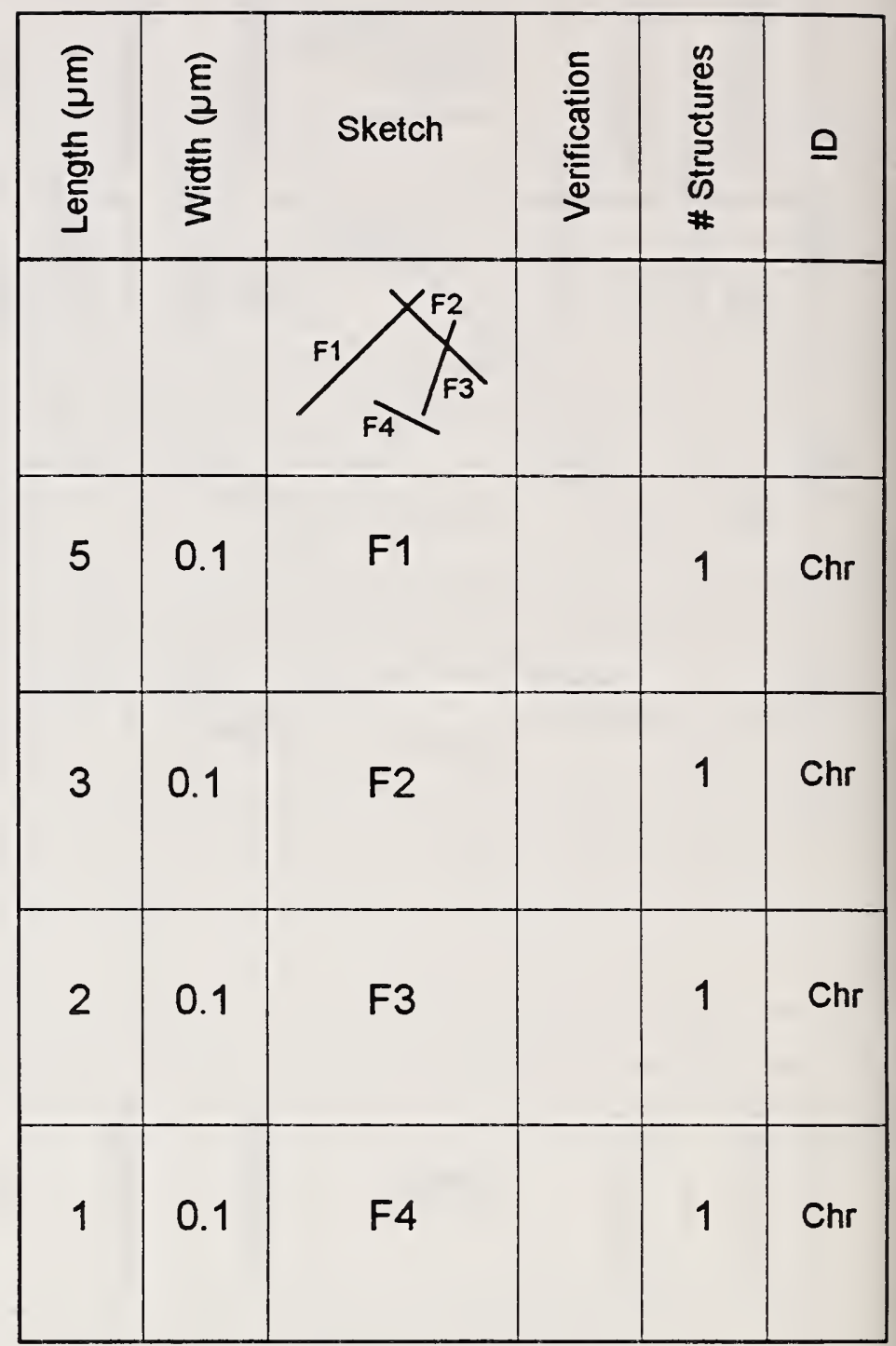

Fig. X2.9 Example of unmatched structures that must be examined by TEM (refer to item 4.5 of the procedure). a) Both analysts have likely found the same particle as indicated by the identification and orientation of the fibers. However, analyst 1 has recorded all fibers as touching (or intersecting) and has therefore counted the fiber arrangement as one structure under the AHERA method. Analyst 2 has reported four structures. The verifying analyst should find and examine the arrangement in the TEM to determine if the fiber labelled as F4 by analyst 2 is touching or intersecting the fiber labelled as F3. b) One possible outcome is that the verifying analyst finds that analyst 1 is correct. Analyst 1 is then assigned a TPMI and analyst 2 is assigned a TPM1 and three FPs. Other possibilities (not illustrated) are that analyst 2 is correct (the structures reported by analyst 2 are then assigned a TPM and 3 TPUs and the structure reported by analyst 1 is assigned a TPM) or that the particle is too contaminated for identification (the structure reported by analyst 1 is then assigned a TPM and those reported by analyst 2 are assigned a TPM and three AMBs). 
Analyst 1

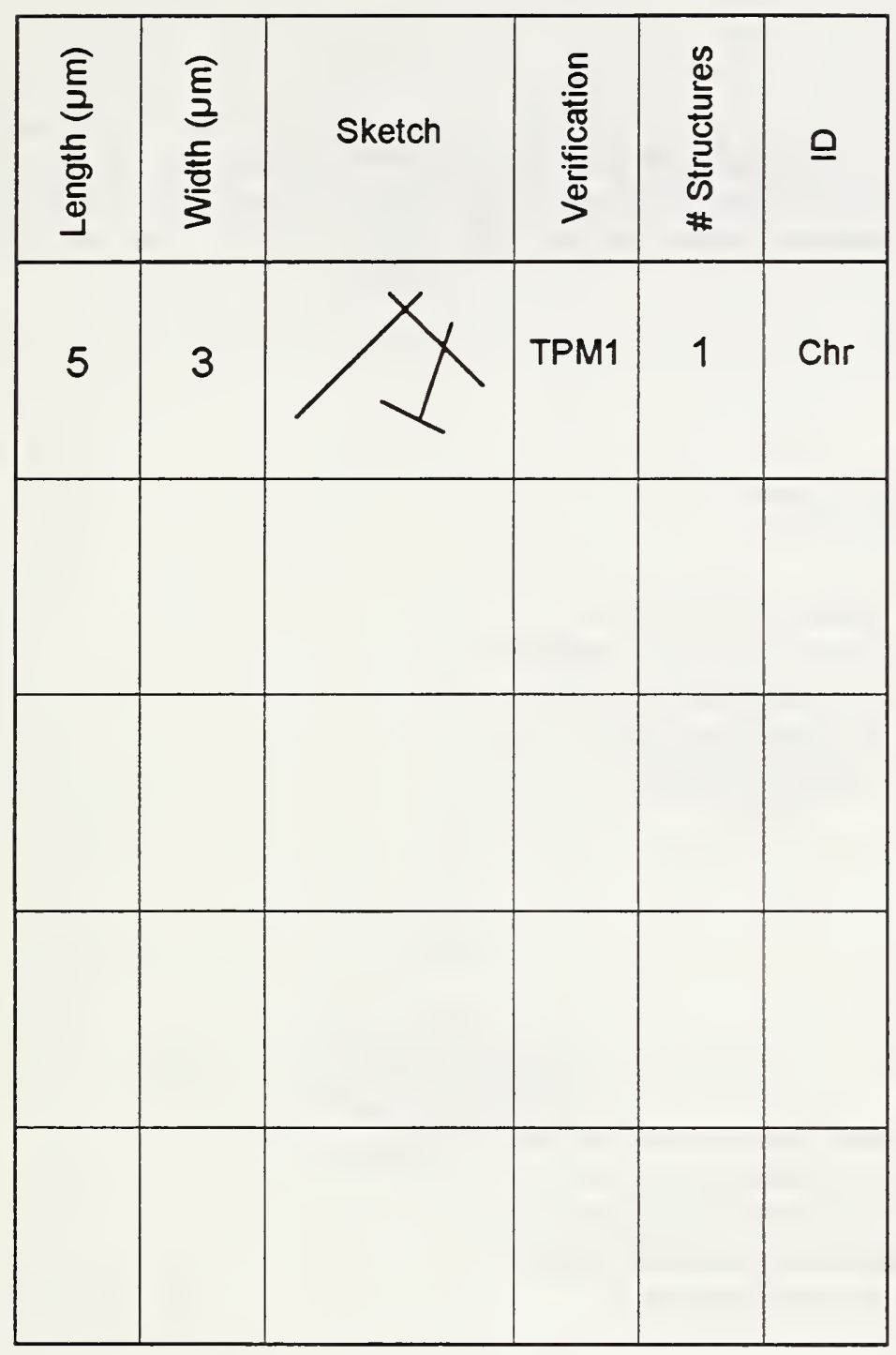

Analyst 2

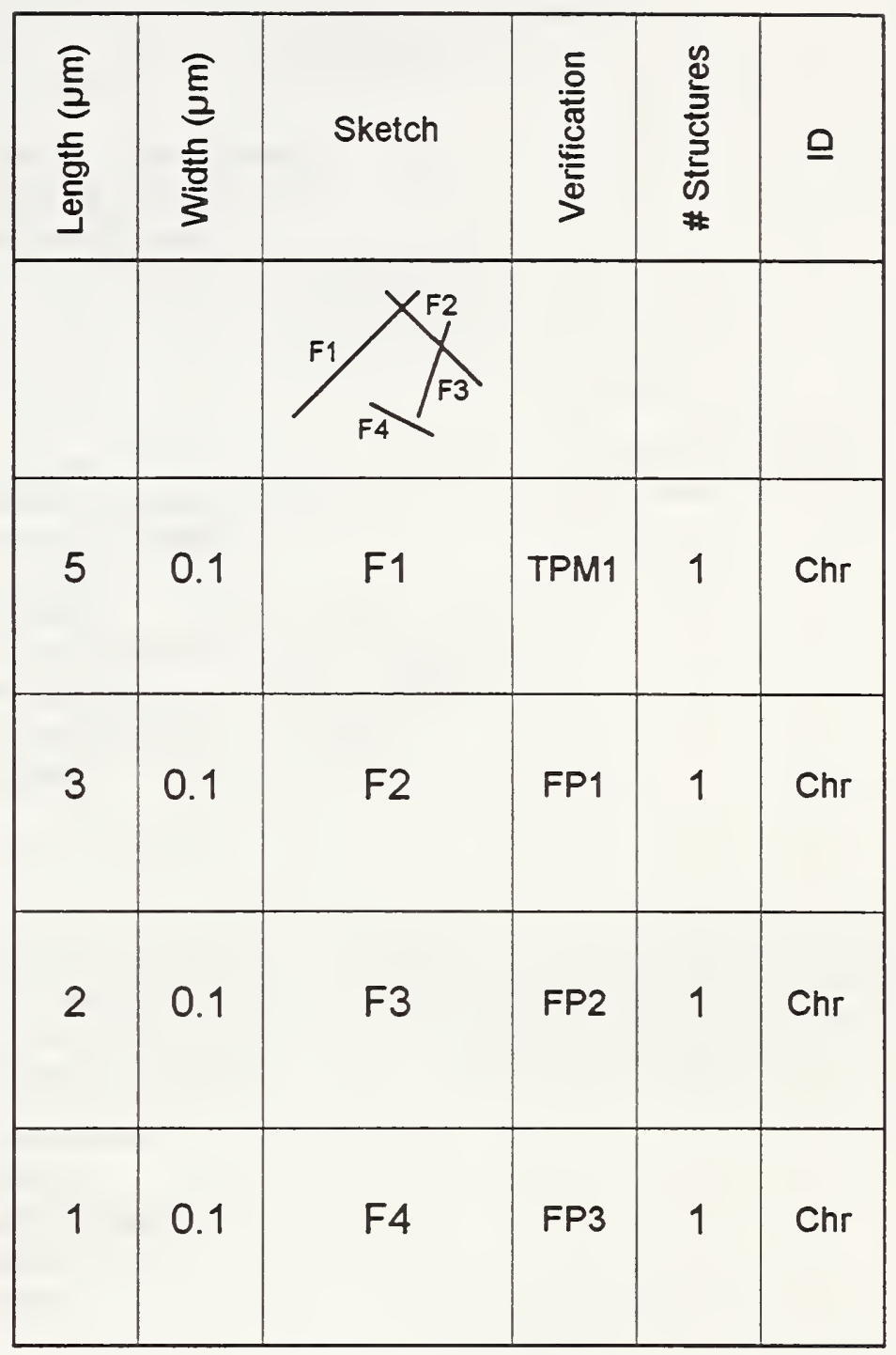

Fig. X2.9 (caption on previous page) 


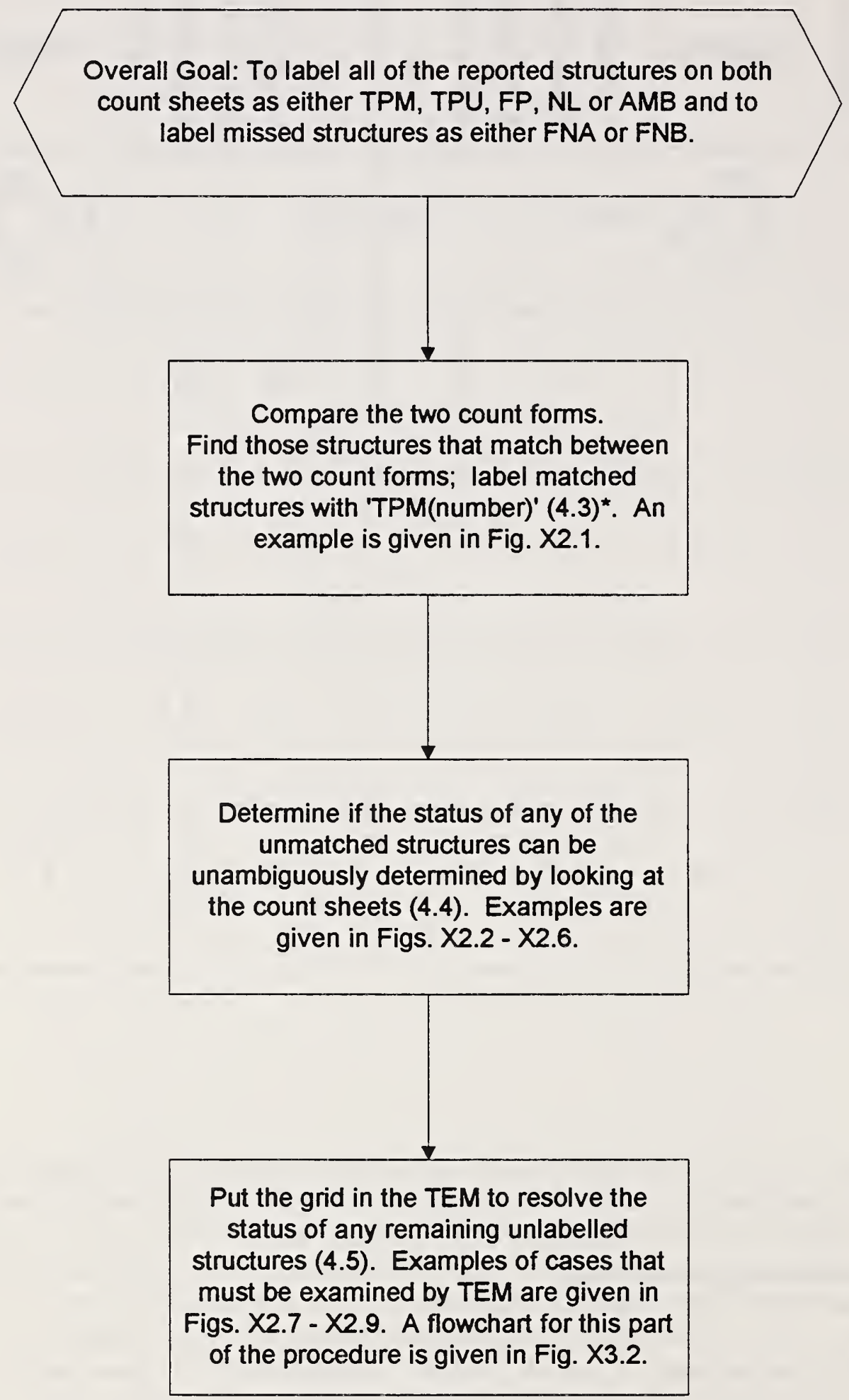

Fig. X3.1 Summary of the overall procedure for comparison of TEM analysis forms by the verifying analyst. *Numbers in parentheses in each block refer to the item number in the procedure. 
Note: if a new structure is identified by the verifying analyst, the structure should be drawn on a new count form and labelled 'TPV(number)'. A label of either 'FNA(number)' or 'FNB(number)' should be put on the two analysts' count forms at the appropriate location (4.6).

Examine the grid square in the TEM.

For each reported structure do the following procedure until all structures are labelled $(4.5)^{*}$.
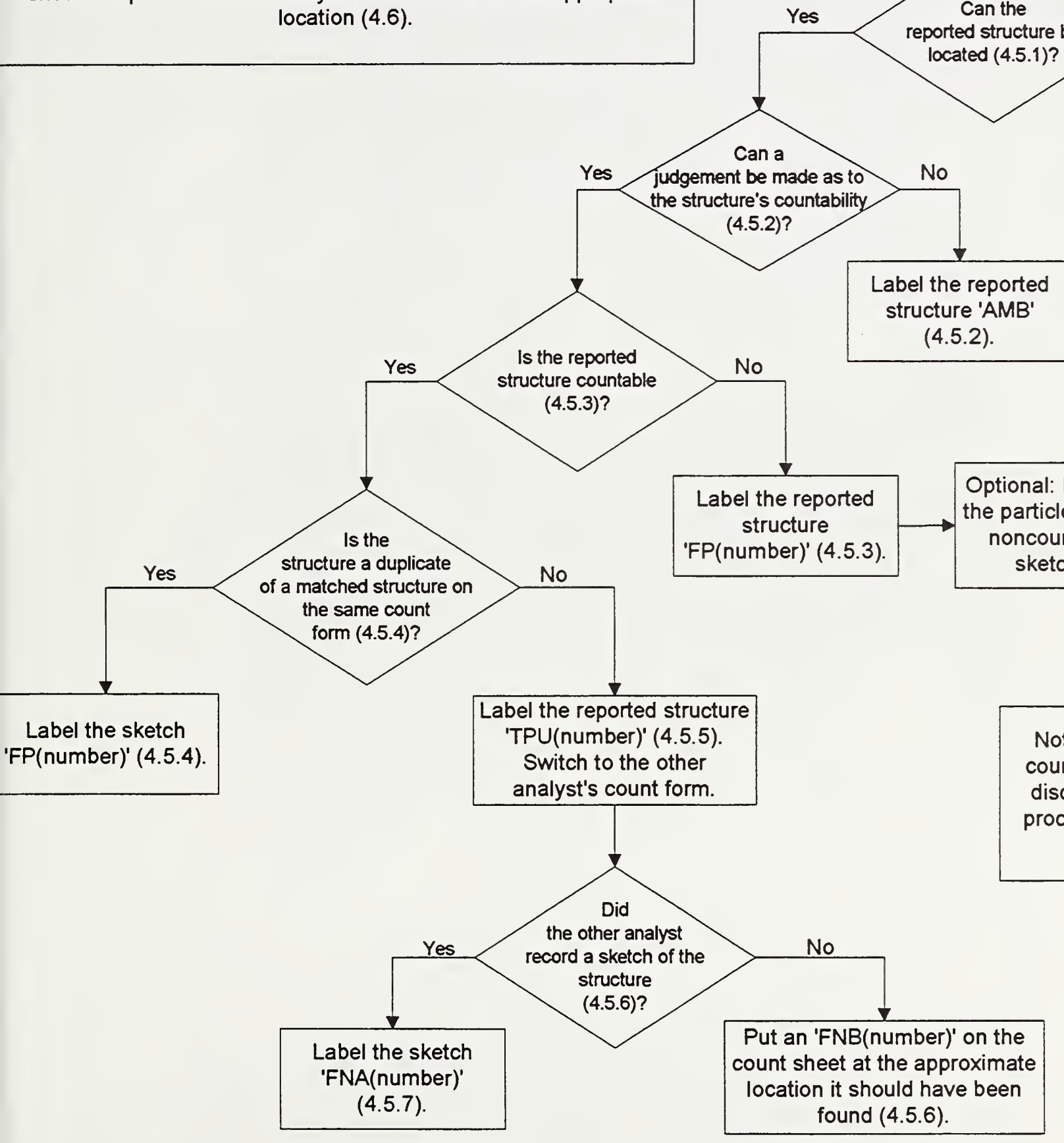

Fig. X3.2 Flowchart for examination of a structure in the TEM. The flowchart is an expansion of the last block in Fig. X3.1. *Numbers in parentheses in each block refer to the item number in the procedure. 


